\title{
Quantum geometry and quantum dynamics at the Planck scale
}

\author{
Martin Bojowald \\ Institute for Gravitation and the Cosmos, The Pennsylvania State University, \\ 104 Davey Lab, University Park, PA 16802, USA
}

\begin{abstract}
Canonical quantum gravity provides insights into the quantum dynamics as well as quantum geometry of space-time by its implications for constraints. Loop quantum gravity in particular requires specific corrections due to its quantization procedure, which also results in a discrete picture of space. The corresponding changes compared to the classical behavior can most easily be analyzed in isotropic models, but perturbations around them are more involved. For one type of corrections, consistent equations have been found which shed light on the underlying spacetime structure at the Planck scale: not just quantum dynamics but also the concept of space-time manifolds changes in quantum gravity. Effective line elements provide indications for possible relationships to other frameworks, such as non-commutative geometry.
\end{abstract}

Keywords: Quantum geometry, space-time diffeomorphisms, cosmological evolution

PACS: 04.60.Ds, 04.60.Pp

\section{SPACE-TIME STRUCTURE}

Canonical formulations provide insights in underlying symmetries, which for gravity correspond to general covariance. Once quantized, correction terms result which may change the underlying symmetries or even provide new quantum degrees of freedom. In loop quantum gravity, corrections arise from quantum geometry (the spatial structure) as well as quantum dynamics. The main recent developments to be described here, obtained from model systems or perturbations, are (i) consistent deformations of classical gravity, and (ii) effective descriptions to derive interacting quantum states and quantum corrections in equations of motion.

\section{Canonical gravity}

For gravity, we have an infinite dimensional phase space of fields $q_{a b}$ (the spatial metric) and momenta $p^{a b}$ (related to extrinsic curvature). The other components of the space-time metric, lapse $N$ and shift $N^{a}$ in

$$
\mathrm{d} s^{2}=g_{\mu \nu} \mathrm{d} x^{\mu} \mathrm{d} x^{v}=-N^{2} \mathrm{~d} t^{2}+q_{a b}\left(\mathrm{~d} x^{a}+N^{a} \mathrm{~d} t\right)\left(\mathrm{d} x^{b}+N^{b} \mathrm{~d} t\right),
$$

are not dynamical since $\dot{N}$ and $\dot{N}^{a}$ do not occur in the action. They may be included in an extended phase space, but their momenta $p_{N}=\delta S / \delta \dot{N}$ and $p_{N^{a}}=\delta S / \delta \dot{N}^{a}$ would be constrained to vanish identically. Accordingly, $\dot{p}_{N}=-\delta S / \delta N$ and $\dot{p}_{N^{a}}=-\delta S / \delta N^{a}$ 
must vanish, too, which implies additional constraints

$$
C=\frac{\sqrt{\operatorname{det} q}}{16 \pi G}^{(3)} R-\frac{16 \pi G}{\sqrt{\operatorname{det} q}}\left(p_{a b} p^{a b}-\frac{1}{2}\left(p_{a}^{a}\right)^{2}\right)=0 \quad, \quad C_{a}=2 D_{b} p_{a}^{b}=0
$$

for the non-trivial phase space variables.

In addition to constraining the fields and their initial values, the constraints generate gauge transformations which must leave physical observables unchanged. The diffeomorphism constraint $D\left[N^{a}\right]=\int \mathrm{d}^{3} x N^{a} C_{a}$ generates spatial diffeomorphisms along a vector field $N^{a}$, while the Hamiltonian constraint $H[N]=\int \mathrm{d}^{3} x N C$ completes this to space-time transformations (for fields satisfying the constraints).

The dynamics of general relativity in a canonical formulation is determined completely by the constraints, forming a total constraint $T\left[N, N^{a}\right]=\int \mathrm{d}^{3} x\left(N C+N^{a} C_{a}\right)=0$ for all multiplier functions $N, N^{a}$. It generates equations of motion $\dot{f}=\left\{f, T\left[N, N^{a}\right]\right\}$ for any phase space function $f(q, p)$, a dot referring to the time gauge as given by the choice of lapse $N$ and shift $N^{a}$ to be inserted in $T\left[N, N^{a}\right]$.

An important consistency requirement follows from this setup: if the constraints must vanish at all times, the time derivative $\dot{T}\left[M, M^{a}\right]=\left\{T\left[M, M^{a}\right], T\left[N, N^{a}\right]\right\}=0$ must vanish for all $N$ and $N^{a}$. The right part of this equation,

$$
\left\{T\left[M, M^{a}\right], T\left[N, N^{a}\right]\right\}=0 \quad \text { if } \quad T\left[N, N^{a}\right]=0,
$$

implies that the constraints form a so-called first class algebra under Poisson brackets. By general covariance, this is automatically satisfied classically: the constraints of general relativity are covariant under space-time diffeomorphisms. As an important consistency condition, (1) must be realized also after quantum corrections have been included in $T\left[N, N^{a}\right]$. If it remains satisfied, an anomaly-free version of quantum effects has been achieved. Since this condition is very restrictive, the anomaly-problem remains one of the most important issues in (canonical) quantum gravity.

If we look at the constraint algebra for general phase space configurations, not just for fields satisfying the constraints, more information about space-time structure can be obtained. Working out the specific constraint algebra for gravity, we obtain

$$
\left\{H\left[N_{1}\right], H\left[N_{2}\right]\right\}=D\left[q^{a b}\left(N_{1} \partial_{b} N_{2}-N_{2} \partial_{b} N_{1}\right)\right]
$$

for the bracket of two Hamiltonian constraints (while brackets involving $D\left[N^{a}\right]$ directly reflect the action of spatial diffeomorphisms on $N$ or $N^{a}$ ). This clearly vanishes once the diffeomorphism constraint is satisfied, but the structure for general fields gives us a wider perspective on the types of gauge transformations involved. If one looks at other generally covariant systems, not just general relativity but also modified versions such as those including extra fields or higher curvature terms, one finds the same constraint algebra. The constraints certainly change, and so does the dynamics, but they still satisfy the same algebra as shown above. The algebra is thus very basic, depending not on the dynamics of the theory but only on the space-time structure. In fact, one can interpret it as generating transformations which correspond to deformations of the hypersurfaces underlying the foliation used to set up the canonical formalism. Accordingly, it is called hypersurface deformation algebra, illustrated in Fig. 1. 


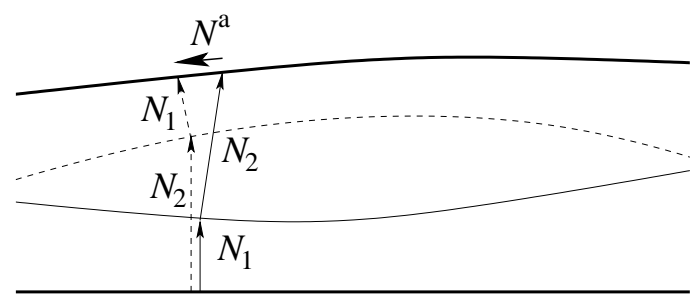

FIGURE 1. Illustration of the hypersurface deformation algebra.

The advantage of using the constraints to generate space-time transformations, rather than Lie derivatives along space-time vector fields, is that this procedure directly deals with objects available after quantization. Neither the manifold nor coordinates will be related to operators, or be represented otherwise in the resulting quantum theory. Their form would have to come out much more indirectly in canonical quantum gravity, but the constraints and their algebra is one of the most basic and important aspects. Although consistently implementing them is certainly not easy, they give us a more direct handle on aspects of quantum space-time.

\section{Quantum corrections}

Canonical quantization following the Dirac procedure turns the constraints into operators and requires them to annihilate physical states. A well-defined quantization is often based on specific constructions which lead to terms in the constraints not seen classically. One, spatial discreteness, is a consequence of some approaches to quantum gravity and leads to deviations from the continuum expressions. After quantization, the constraints typically change, and so does their algebra. In this way, one can analyze consequences for the quantum space-time structure without direct reference to manifolds or coordinates, which would no longer be part of the quantum theory.

Specifically, there are three types of corrections in loop quantum gravity [1, 2, 3], which in general are equally important:

- Entire states evolve which spread and deform. Quantum fluctuations, correlations and higher moments are independent variables back-reacting on expectation values. This is well-known from quantum mechanics and quantum field theory, where its effects can be captured by the loop expansion.

- In loop quantum gravity, holonomies

$$
h_{e}(A)=\mathscr{P} \exp \left(\int_{e} A_{a}^{i} \tau_{i} \mathrm{~d} t\right)
$$

as non-local, non-linear functions imply higher order corrections when they appear in constraints in place of the connection $A_{a}^{i}$ [4].

- Again in loop quantum gravity, fluxes

$$
F_{S}(E)=\int_{S} \mathrm{~d}^{2} y E_{i}^{a} n_{a}
$$


quantizing the spatial metric have discrete spectra containing zero. Inverse metric components receive corrections for discrete (lattice-like) states with small elementary areas [5].

Canonical methods for the first class of effects are available [6], including the treatment of constraints [7, 8] (but net yet fully extended to fields). The latter two directly probe quantum geometry, intimately tied to the discreteness.

\section{QUANTUM FRIEDMANN EQUATION}

Several of these effects can be illustrated easily in isotropic cosmological models. In this case, they result in a corrected Friedmann equation which can be summarized as

$$
\left(\frac{\dot{a}}{a}\right)^{2}=\frac{8 \pi G}{3}\left(\rho\left(1-\frac{\rho_{Q}}{\rho_{\text {crit }}}\right)+\frac{1}{2} \sqrt{1-\frac{\rho_{Q}}{\rho_{\text {crit }}}} \eta(\rho-P)+\frac{(\rho-P)^{2}}{(\rho+P)^{2}} \eta^{2}\right)
$$

where $P$ is pressure in addition to the energy density $\rho$ [9]; for earlier versions see $[10,11,12,13]$. Quantum effects thus make the Friedmann equation pressure dependent, which would not appear in the classical equation. Moreover, there are corrections depending on the form of the quantum state (of gravity, not matter). First, $\eta$ parameterizes quantum correlations; it would vanish only for a completely uncorrelated state. Secondly, quantum fluctuations, or a whole series of fluctuation parameters $\varepsilon_{k}$ obtained from higher moments of the state, define

$$
\rho_{Q}:=\rho+\varepsilon_{0} \rho_{\text {crit }}+(\rho-P) \sum_{k=0}^{\infty} \varepsilon_{k+1}(\rho-P)^{k} /(\rho+P)^{k}
$$

This corrected, quantum density appears in a term together with the critical density $\rho_{\text {crit }}=3 / 8 \pi G L(a)^{2}$, which does not introduce a new degree of freedom but depends on a characteristic length scale $L(a)$ used in setting up the reduced model. (The size of this parameter and its possible $a$-dependence are currently not fixed, but should in principle be derived from a full theory once the underlying state is under control. Till then, constraints on its value can be found by internal consistency or phenomenology.)

In general, the dynamics is complicated since not only $a$ or the matter field would be dynamical, but also the moments contained in $\eta$ and $\rho_{Q}$ : The entire state evolves, not just its expectation values giving rise to $a$ and the classical matter variables. We are forced to deal with a higher-dimensional effective system, containing new quantum degrees of freedom. However, there are cases in which the behavior simplifies. If $\rho=P$, for instance, which is realized for a free, massless scalar, the state parameters decouple: the model becomes exactly solvable and free of quantum back-reaction [14, 15]. Then, the only correction to the Friedmann equation is to replace $\rho$ with $\rho\left(1-\rho / \rho_{\text {crit }}\right)$, implying bouncing solutions [13]. This special model was in fact first studied in considerable detail, mainly by numerically solving for the wave function [16]. A similar equation results when $\eta=0$ around the point where $\rho_{Q}=\rho_{\text {crit }}$, but this seems to require more special properties of the state. 
The main origin of corrections giving rise to bouncing solutions is the use of holonomies. By reformulating the higher order terms which they initially imply for $\dot{a}$, they give rise to the quadratic energy term in the Friedmann equation (see e.g. [17]). To understand all implications of the bounce for cosmology, including metric perturbations at least at the linear level would be essential. Unfortunately, so far no consistent inhomogeneous formulation with holonomy corrections is available. Available equations either apply only to special modes [18, 19], or are based on gauge fixing [20, 21]. In the latter case, anomalies or crucial quantum effects are hidden, but would have important consequences for the behavior. (Examples are mentioned below.) It thus remains unclear how bounces as they can sometimes be obtained from effective Friedmann equations fit into a cosmological scenario. Consistent equations for inhomogeneities in the presence of corrections from loop quantum gravity are, however, available for inverse metric corrections, to which we turn now. This brings us back to the structure of space-time in canonical quantum gravity.

\section{INVERSE METRIC CORRECTIONS}

Inverse metric corrections change the Hamiltonian constraint, in its gravitational part [22] as well as matter Hamiltonians [5], whenever inverse components of the densitized triad appear. To avoid details and focus on generic implications, we can implement them by a generic function $\alpha$ depending on the gravitational phase space variables. In some cases, such as isotropic models [23, 24] or regular lattice states with gauge fixing [25] the form can be computed explicitly, although it remains subject to quantization ambiguities; see Fig. 2,

\section{Number of spatial atoms}

Quantum geometry corrections of loop quantum gravity depend on the form and size of discrete building blocks realized in the theory and its states. Geometrical operators have discrete spectra [26, 27, 28], showing that the spatial geometry is made up from small constituents. These constituents come in different sizes, determined by the spin labels of a spin network state, and can form a macroscopic geometry in many different ways. Dynamically, one expects the constituents to change in size as well as number, giving rise to the evolution of a continuous geometry on large scales.

Specifically, this is realized in loop quantum cosmology by the mathematical objects of holonomies (3) and fluxes (4), whose elementary size in terms of coordinates we call $\ell_{0}$ (linear for holonomies, quadratic for fluxes). In a region of total coordinate volume $V_{0}$, the number of "atoms of geometry" is then $\mathscr{N}=V_{0} / \ell_{0}^{3}$. This parameter depends on the size of the region chosen, but not on coordinates. The density $\mathscr{N} / V_{0}=\ell_{0}^{-3}$, on the other hand, is independent of the region but coordinate dependent. The only coordinate and region-independent measure for the denseness of spatial atoms is the geometrical density $\rho_{\mathscr{N}}=\mathscr{N} / a^{3} V_{0}=\left(\ell_{0} a\right)^{-3}$ in a universe of scale factor $a$. Although $\ell_{0}$ is fixed for basic operators in static quantum geometry, before the constraints are imposed, capturing the full dynamics of changing lattices requires $\ell_{0}$ to depend on "time" [29]. This is to 
be understood in the internal time sense, e.g. with reference to the scale factor: $\ell_{0}(a)$. The same function determines the characteristic scale $L$ seen in the critical density for Friedmann universes: $L(a)=a \ell_{0}(a)$.

These parameters enter basic holonomies via $\exp \left(i \ell_{0} \dot{a} / N\right)$ (with the lapse function $N$, which is one if the dot refers to proper time) and fluxes via $\mu=\ell_{0}^{2} a^{2} / \ell_{\mathrm{P}}^{2}$ if the geometry is nearly isotropic. Strong quantum corrections (holonomies deviating strongly from $\dot{a} / N$, or large inverse metric corrections $\alpha(\mu)$ ) result in both cases if the arguments $\ell_{0} \dot{a} / N$ of holonomies or the values of fluxes are of the order one. Holonomy corrections thus appear when the curvature is $\dot{a} / N \sim k_{*}:=\left(\mathscr{N} / V_{0}\right)^{1 / 3}$. Inverse metric corrections are large when $a \sim a_{*}:=\left(\mathscr{N} / V_{0}\right)^{1 / 3} \ell_{\mathrm{P}}$. (Notice that these equations are coordinate inpendent even though each side of the inequalities depends on coordinates. For instance, for $a \sim a_{*}$, the geometrical vertex density $\mathscr{N} / a^{3} V_{0}=\left(a_{*} / a\right)^{3} / \ell_{\mathrm{P}}^{3}$ is near one per Planck volume, a statement completely independent of coordinates as well as $V_{0}$ or any region chosen.) The classical range, when all corrections are small, is characterized by $\dot{a} / N \ll$ $k_{*}$ and $a \gg a_{*}$, as determined by the vertex density $\mathscr{N} / V_{0}$ of a quantum geometry state.

For cosmological evolution, the dependence of $\mathscr{N}$ on $a$ is important, which occurs whenever the discrete structure is being refined during expansion: spatial atoms emerge dynamically, ensuring that the discrete geometry is not enlarged to macroscopic sizes by cosmic expansion. A derivation of this refinement from a full theory of quantum gravity, including all inhomogeneities, is challenging. At the current stage, this picture gives rise to different models obtained by parameterizations. If one assumes a power

law $\mathscr{N} \propto a^{-6 x}$, which can describe at least finite ranges of evolution, one generically expects $-1 / 2<x<0$ according to what is known about the dynamics of loop quantum gravity. The limiting cases are interpreted as follows: For $x=0, \mathscr{N}$ is constant and there is no refinement; discrete building blocks are just enlarged during expansion. As one may expect, this leads to late-time problems especially during the prolonged expansion of inflation [30, 31]. For $x=-1 / 2$, on the other hand, we have a constant size of building blocks, and their number increases proportionally to volume. There are no further excitations of spatial "atoms" beyond their initial size.

Such effects, though schematic, have surprisingly strong consequences. First, consistency bounds from the interplay of holonomy and inverse metric corrections [32] or from more restrictive anisotropic models [33] exist. Secondly, this allows us to use phenomenology to see how quantum gravity dynamically refines its discrete space. Recent examples have resulted, for instance, in an upper bound $\mathscr{N} / a^{3} V_{0}<3 / \ell_{\mathrm{P}}^{3}$ for the density from big bang nucleosynthesis [34], or a characteristic blue-tilt for tensor modes which is enhanced if $x>-1 / 2$ while for $x=-1 / 2$ correction are only small and of size $8 \pi G \rho \ell_{\mathrm{P}}^{2}[35,36]$.

\section{Linear perturbations}

Once a fully consistent theory for linear perturbations, including inverse metric corrections, is set up and evaluated, restrictions on quantum geometry might become even sharper. For linear metric perturbations around Friedmann-Robertson-Walker backgrounds (which require the Hamiltonian to be expanded to second order) the corrected 


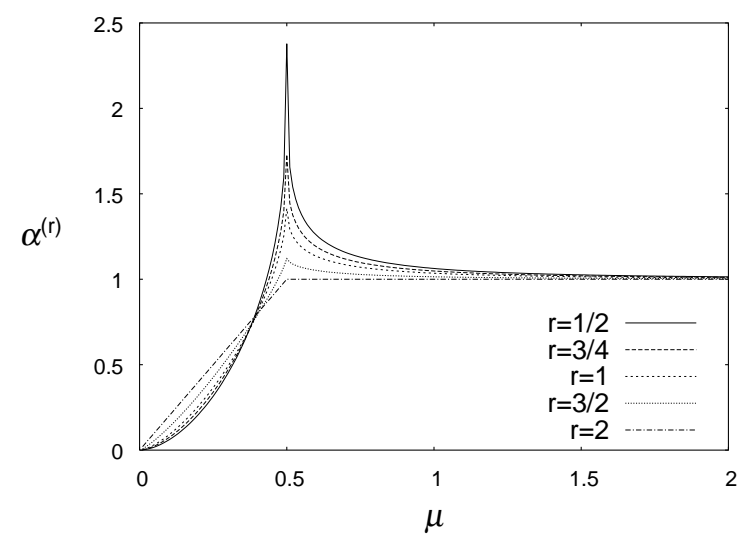

FIGURE 2. Examples for inverse metric correction functions, subject to ambiguities parameterized by $0<r \leq 2$.

Hamiltonian can be expanded as [37]

$$
H_{\text {grav }}^{Q}:=\frac{1}{16 \pi G} \int \mathrm{d}^{3} x\left(\bar{N}\left(\bar{\alpha} \mathscr{H}^{(0)}+\alpha^{(2)} \mathscr{H}^{(0)}+\bar{\alpha} \mathscr{H}^{Q(2)}\right)+\delta N \bar{\alpha} \mathscr{H}^{Q(1)}\right)
$$

with $\mathscr{H}^{(0)}=-6 \mathscr{H}^{2} a$ (the background Hamiltonian) and

$$
\begin{aligned}
\mathscr{H}^{Q(1)}= & -4(1+f) \mathscr{H} a \delta_{j}^{c} \delta K_{c}^{j}-(1+g) \frac{\mathscr{H}^{2}}{a} \delta_{c}^{j} \delta E_{j}^{c}+\frac{2}{a} \partial_{c} \partial^{j} \delta E_{j}^{c} \\
\mathscr{H}^{Q(2)}= & a \delta K_{c}^{j} \delta K_{d}^{k} \delta_{k}^{c} \delta_{j}^{d}-a\left(\delta K_{c}^{j} \delta_{j}^{c}\right)^{2}-\frac{2 \mathscr{H}^{a}}{a} \delta E_{j}^{c} \delta K_{c}^{j} \\
& -\frac{\mathscr{H}^{2}}{2 a^{3}} \delta E_{j}^{c} \delta E_{k}^{d} \delta_{c}^{k} \delta_{d}^{j}+\frac{\mathscr{H}^{2}}{4 a^{3}}\left(\delta E_{j}^{c} \delta_{c}^{j}\right)^{2}-(1+h) \frac{\delta^{j k}}{2 a^{3}}\left(\partial_{c} \delta E_{j}^{c}\right)\left(\partial_{d} \delta E_{k}^{d}\right) .
\end{aligned}
$$

Here, $\delta E_{i}^{a}$ and $\delta K_{a}^{i}$ are perturbations of the densitized triad and extrinsic curvature, respectively. In addition to the primary correction function $\alpha$, with its background value $\bar{\alpha}$ and the second order contribution $\alpha^{(2)}$, there are extra function $f, g$ and $h$. They will be fixed in terms of $\alpha$ later on.

Modifying the constraints in most cases leads to anomalies: classical first class constraints then no longer form a first class algebra. Severe consistency issues result, such as inconsistent equations or the coupling of gauge parameters to observables. For a gauge system, quantization or any form of quantum corrections of the constraints must be a consistent deformation, respecting the first class nature of the constraints. For constraints corrected by inverse triad corrections as above, a first class algebra to second order in perturbations is realized if the correction functions satisfy $2 f+g=0$ and

$$
\begin{gathered}
-h-f+\frac{a}{\bar{\alpha}} \frac{\partial \bar{\alpha}}{\partial a}=0 \quad, \quad f-g-2 a \frac{\partial f}{\partial a}-\frac{a}{\bar{\alpha}} \frac{\partial \bar{\alpha}}{\partial a}=0 \\
\frac{1}{6} \frac{\partial \bar{\alpha}}{\partial a} \frac{\delta E_{j}^{c}}{a^{3}}+\frac{\partial \alpha^{(2)}}{\partial\left(\delta E_{i}^{a}\right)}\left(\delta_{j}^{a} \delta_{i}^{c}-\delta_{j}^{c} \delta_{i}^{a}\right)=
\end{gathered}
$$


(There are additional conditions for matter correction functions in terms of $\bar{\alpha}^{2}$ if matter is present.)

With these equations, all initial coefficients are fixed in terms of $\bar{\alpha}$, whose general form can be derived in models. Importantly, corrections of inverse metric type are possible in a consistent deformation: $\bar{\alpha}$ remains undetermined from the algebra, and so need not take the classical value $\bar{\alpha}=1$. It is allowed to be of the form seen in Fig. 2 , for instance. Inverse metric corrections can be implemented in an anomaly-free form, producing consistent equations for scalar linear perturbations [38],

$$
\partial_{c}(\dot{\Psi}+\mathscr{H}(1+f) \Phi)=\pi G \frac{\bar{\alpha}}{\bar{v}} \dot{\bar{\varphi}} \partial_{c} \delta \varphi^{\mathrm{GI}}
$$

from the diffeomorphism constraint,

$$
\begin{aligned}
& \Delta\left(\bar{\alpha}^{2} \Psi\right)-3 \mathscr{H}(1+f)(\dot{\Psi}+\mathscr{H} \Phi(1+f)) \\
= & 4 \pi G \frac{\bar{\alpha}}{\bar{v}}\left(1+f_{3}\right)\left(\dot{\bar{\varphi}} \delta \dot{\varphi}^{\mathrm{GI}}-\dot{\bar{\varphi}}^{2}\left(1+f_{1}\right) \Phi+\bar{v} a^{2} V_{, \varphi}(\bar{\varphi}) \delta \varphi^{\mathrm{GI}}\right)
\end{aligned}
$$

from the Hamiltonian constraint, and

$$
\begin{aligned}
& \ddot{\Psi}+\mathscr{H}\left(2 \dot{\Psi}\left(1-\frac{a}{2 \bar{\alpha}} \frac{\mathrm{d} \bar{\alpha}}{\mathrm{d} a}\right)+\dot{\Phi}(1+f)\right)+\left(2 \dot{\mathscr{H}}+\mathscr{H}^{2}\left(1+\frac{a}{2} \frac{\mathrm{d} f}{\mathrm{~d} a}-\frac{a}{2 \bar{\alpha}} \frac{\mathrm{d} \bar{\alpha}}{\mathrm{d} a}\right)\right) \Phi(1+f) \\
& =4 \pi G \frac{\bar{\alpha}}{\bar{v}}\left(\dot{\bar{\varphi}} \delta \dot{\varphi}^{\mathrm{GI}}-a^{2} \bar{v} V_{, \varphi}(\bar{\varphi}) \delta \varphi^{\mathrm{GI}}\right)
\end{aligned}
$$

as the equation of motion. They show promising effects not appearing classically, for instance the non-conservation of power on large scales. Another implication is the existence of anisotropic stress, a consequence not seen in gauge-fixed treatments: with the corrections, $\Phi=(1+h) \Psi$.

\section{Quantum constraint algebra}

With the required conditions for $f, g$ and $h$ and $\alpha^{(2)}$, the algebra of corrected constraints is first class: it presents a consistent deformation of the classical theory to linear order in inhomogeneities. Anomaly-free constraints including quantum gravity corrections thus exist. Even though the underlying discreteness, via inverse metric corrections, is responsible for the occurrence of these corrections, it does not destroy general covariance. [37]:

However, the constraint algebra of hypersurface deformations is quantum corrected

$$
\left\{H^{Q}\left[N_{1}\right], H^{Q}\left[N_{2}\right]\right\}=D\left[\bar{\alpha}^{2} \bar{N} a^{-1 / 2} \partial^{a}\left(\delta N_{2}-\delta N_{1}\right)\right] .
$$

(The same corrected algebra results in spherically symmetric models without linearization [39, 40].) This may not be fully surprising since the classical algebra (2) contains the inverse metric in its structure functions, and so inverse metric corrections may be 
expected in the constraint algebra. What is non-trivial is the conclusion that this correction can be implelented anomaly-freely. The specific form obtained here indicates how the structure of quantum space-time changes compared to the classical one: even the constraint algebra, and thus the underlying algebra of space-time diffeomorphisms, is corrected. Quantum gravity corrections affect not only the dynamics of the theory, but also its underlying symmetries. An immediate consequence in a canonical theory is that quantum corrections to constraints change the form of gauge invariant variables, as they appear in the consistent perturbation equations presented before. Here, differences to reduced phase space quantizations arise, where classical gauge invariant quantities would be quantized directly without implementing corrections to the gauge behavior.

While it is clear that the quantum space-time structure must change from inverse metric corrections, it is difficult to say what the new manifold structure might be. We only know the constraint algebra so far, which is difficult to integrate. Moreover, we only know the corrections for linear perturbative inhomogeneities, and an extension to higher orders or non-perturbative inhomogeneity is much more involved. (But as suggested by non-linear spherically symmetric models, it may well be possible.) An intriguing possibility, still to be explored, would be a relationship to non-commutative geometry. In both cases, canonical quantum gravity and non-commutative geometry, manifolds are not taken as basic. But effective structures do arise, which may be the best way to compare these different frameworks. If such a relationship can be established, it might give indications for deformed Lorentz symmetries in quantum gravity; see e.g. [41].

\section{CONCLUSIONS}

Different types of quantum corrections arise in loop quantum gravity: those from quantum geometry (inverse metric/holonomy corrections) and those from quantum dynamics (back-reaction). The former are specific to the theory and thus provide useful opportunities for tests.

In particular the anomaly problem, which becomes severe in the context of inhomogeneities and in particular with discreteness corrections, can be addressed at an effective level. It turns out that consistent deformations do exist, incorporating quantum effects from the inverse metric (themselves coming from discrete flux spectra) in classical equations. Via the consistent perturbation equations, an interface to cosmological applications is obtained. Observational input is very conceivable, and can shed light on the underlying quantum states by constraining possible quantum corrections.

On a fundamental level, this tells us that discrete structures of space-time do not have to break covariance. They may deform the classical algebra, but the same number of symmetry generators remains present. A different realization of covariance results, perhaps as a deformed space-time diffeomorphism group.

\section{ACKNOWLEDGMENTS}

Work reported here was supported in part by NSF grant PHY-0748336. 


\section{REFERENCES}

1. C. Rovelli, Quantum Gravity, Cambridge University Press, Cambridge, UK, 2004.

2. A. Ashtekar, and J. Lewandowski, Class. Quantum Grav. 21, R53-R152 (2004), gr-qc/ 0404018.

3. T. Thiemann, Introduction to Modern Canonical Quantum General Relativity, Cambridge University Press, Cambridge, UK, 2007, gr-qc/0110034.

4. C. Rovelli, and L. Smolin, Nucl. Phys. B 331, 80-152 (1990).

5. T. Thiemann, Class. Quantum Grav. 15, 1281-1314 (1998), gr-qc/9705019.

6. M. Bojowald, and A. Skirzewski, Rev. Math. Phys. 18, 713-745 (2006), math-ph/0511043.

7. M. Bojowald, B. Sandhöfer, A. Skirzewski, and A. Tsobanjan, Rev. Math. Phys. 21, 111-154 (2009), arXiv:0804.3365.

8. M. Bojowald, and A. Tsobanjan, arXiv:0906.1772,

9. M. Bojowald, Phys. Rev. Lett. 100, 221301 (2008), arXiv:0805.1192

10. K. Banerjee, and G. Date, Class. Quant. Grav. 22, 2017-2033 (2005), gr-qc/0501102.

11. G. Date, and G. M. Hossain, Phys. Rev. Lett. 94, 011302 (2005), gr-qc/ 0407074.

12. P. Singh, and K. Vandersloot, Phys. Rev. D 72, 084004 (2005), gr-qc/ 0507029.

13. P. Singh, Phys. Rev. D 73, 063508 (2006), gr-qc/0603043

14. M. Bojowald, Phys. Rev. D 75, 081301(R) (2007), gr-qc/0608100

15. M. Bojowald, Phys. Rev. D 75, 123512 (2007), gr-qc/ 0703144

16. A. Ashtekar, T. Pawlowski, and P. Singh, Phys. Rev. D 73, 124038 (2006), gr-qc/0604013

17. K. Vandersloot, Phys. Rev. D 71, 103506 (2005), gr-qc/ 0502082.

18. M. Bojowald, and G. Hossain, Class. Quantum Grav. 24, 4801-4816 (2007), arXiv: 0709.0872

19. M. Bojowald, and G. Hossain, Phys. Rev. D 77, 023508 (2008), arXiv: 0709.2365 ,

20. M. Artymowski, Z. Lalak, and L. Szulc, JCAP 0901, 004 (2009), arXiv: 0807.0160

21. J. Mielczarek, arXiv:0908.4329

22. T. Thiemann, Class. Quantum Grav. 15, 839-873 (1998),gr-qc/9606089

23. M. Bojowald, Phys. Rev. D 64, 084018 (2001), gr-qc/0105067.

24. M. Bojowald, Class. Quantum Grav. 19, 5113-5130 (2002), gr-qc/0206053.

25. M. Bojowald, H. Hernández, M. Kagan, and A. Skirzewski, Phys. Rev. D 75, 064022 (2007), gr-qc/0611112.

26. C. Rovelli, and L. Smolin, Nucl. Phys. B 442, 593-619 (1995), erratum: Nucl. Phys. B 456, 753 (1995), gr-qc/9411005.

27. A. Ashtekar, and J. Lewandowski, Class. Quantum Grav. 14, A55-A82 (1997), gr-qc/9602046

28. A. Ashtekar, and J. Lewandowski, Adv. Theor. Math. Phys. 1, 388-429 (1998), gr-qc/9711031.

29. M. Bojowald, Gen. Rel. Grav. 38, 1771-1795 (2006), gr-qc/ 0609034.

30. W. Nelson, and M. Sakellariadou, Phys. Rev. D 76, 044015 (2007), arXiv: 0706.0179

31. W. Nelson, and M. Sakellariadou, Phys. Rev. D 76, 104003 (2007), arXiv: 0707.0588

32. M. Bojowald, Class. Quantum Grav. 26, 075020 (2009), arXiv:0811.4129.

33. M. Bojowald, D. Cartin, and G. Khanna, Phys. Rev. D 76, 064018 (2007), arXiv: 0704.1137

34. M. Bojowald, R. Das, and R. Scherrer, Phys. Rev. D 77, 084003 (2008), arXiv:0710.5734.

35. A. Barrau, and J. Grain, arXiv:0805.0356.

36. A. Barrau, and J. Grain, Phys. Rev. Lett. 102, 081301 (2009), arXiv:0902.0145,

37. M. Bojowald, G. Hossain, M. Kagan, and S. Shankaranarayanan, Phys. Rev. D 78, 063547 (2008), arXiv:0806.3929.

38. M. Bojowald, G. Hossain, M. Kagan, and S. Shankaranarayanan, Phys. Rev. D 79, 043505 (2009), arXiv:0811.1572.

39. M. Bojowald, and J. D. Reyes, Class. Quantum Grav. 26, 035018 (2009), arXiv:0810.5119.

40. M. Bojowald, J. D. Reyes, and R. Tibrewala, Phys. Rev. D 80, 084002 (2009), arXiv: 0906.4767

41. J. Kowalski-Glikman, Lect. Notes Phys. 669, 131-159 (2005), hep-th/0405273. 